\title{
CARACTERIZACIÓN DE 1.131 PACIENTES CON CÁNCER CÉRVICO-UTERINO SEGÚN ESTADIO FIGO: HOSPITAL GUILLERMO GRANT, 1999-2005
}

\author{
Jorge Cabrera D. ${ }^{1}$, Juan Villanueva G. ${ }^{1}$, César García Z. ${ }^{2}$, Ángela Fernández S. ${ }^{2}$, \\ Clemencia Cabrera F. ${ }^{1}$, Maritza Flores $0^{3}$
}

\footnotetext{
${ }^{1}$ Departamento y Servicio de Obstetricia y Ginecología, ${ }^{2}$ Servicio de Oncología, Hospital Guillermo Grant B., ${ }^{3}$ Departamento de Salud Pública, Facultad de Medicina, Universidad de Concepción.
}

\section{RESUMEN}

Objetivo: Analizar la distribución de mujeres con diagnóstico histológico de cáncer cérvico-uterino de acuerdo a la etapa clínica y edad. Método: Estudio de corte transversal de 1.131 mujeres clasificadas de acuerdo a la etapa FIGO en el Hospital Guillermo Grant (1999 - 2005). Resultados: 522 casos (46,1\%) corresponden a Etapa 0, con edad promedio de 34,9 años; el 9\% eran menores de 25 años; el 40,6\% se concentró en el grupo entre 25 y 34 años. A cáncer invasor corresponden 609 mujeres (53,9\%), con edad promedio de 51,2 años, en Etapa I el 36,6\%, Etapa II el 31,8\%, Etapa III el 26,1\% y Etapa IV el 1,3\%. Del grupo de mujeres con cáncer invasor, 117 (19,2\%) eran mayores de 65 años y 4 (0,6\%) menores de 25 años. Conclusión: La aplicación del Programa Nacional de pesquisa precoz del cáncer cérvico-uterino hizo que el $46,1 \%$ de los casos correspondan a diagnóstico precoz, contribuyendo a la tendencia nacional de disminución de la tasa de mortalidad por esta patología. Hay una disminución progresiva del número de casos de cáncer invasor a medida que se avanza en las etapas, coincidentemente hay un aumento en la edad de estas mujeres, es así que el $19,2 \%$ eran mayores de 65 años. De acuerdo con estos resultados es necesario analizar el costobeneficio de incluir mujeres menores de 25 años y mayores de 65 en el programa de pesquisa precoz.

\section{PALABRAS CLAVE: Cáncer cérvico uterino, diagnóstico precoz, clasificación FIGO}

\section{SUMMARY}

Objective: To analyze the distribution according to stage and age of women with histological cervical carcinoma confirmed diagnosis. Method: A cross sectional study of 1,131 women of the Hospital Guillermo Grant B with FIGO's classification, in the period 1999-2005. Results: From the 1,131 cases, $522(46.1 \%)$ women correspond to Stage 0, the average age was 34.9 years; $9 \%$ were younger than 25 years of age; $40.6 \%$ were between 25-34 years old. Invasive cancer had 609 women (53.9\%), average age was 51.2 years, corresponding $223(36.6 \%)$ Stage I, 194 (31.8\%) Stage II, 159 (26.1\%) Stage III, and 8 (1.3\%) Stage IV. The range of age in invasive stages was between 19-95 years. From the group of women with cancer, 117 $(19.2 \%)$ were older than 65 years, and 4 women $(0.6 \%)$ were younger than 25 . Conclusion: The implementation of the National Program of early detection for cervical cancer caused that $46.1 \%$ of cases correspond to early diagnosis, contributing to the national trend of decreasing rate of mortality for this disease. There is a progressive decrease in the incidence of invasive cancer as it progresses in stages, coincidentally there is an increase in the age of these women, so that $19.2 \%$ were older than 65 years. Based on these results it is necessary to analyze the cost benefit of including women younger than 25 years and older than 65 in the screening.

KEY WORDS: Cervical carcinoma, early diagnosis, FIGO classification 


\section{INTRODUCCIÓN}

Los programas de pesquisa del cáncer cérvico-uterino (CACU), aseguran una disminución de la incidencia de nuevos casos y la consecuente disminución de la mortalidad, contribuyendo así al aumento de la supervivencia $(1,2)$. En Chile, la tasa de mortalidad por CACU ha disminuido en forma sostenida. La última cifra promedio correspondiente al año 2005 es de 8,2 por 100.000 mujeres, conformada por tasas muy disímiles de las diferentes regiones $(3,4,5)$.

Este éxito relativo se debe, entre otros, a las nuevas exigencias del programa, al aumento de la cobertura, al manejo más ágil en la Unidad de Patología Cervical (UPC), al alto índice de curación de las lesiones intraepiteliales de alto grado $(6,7,8)$, al éxito del tratamiento quirúrgico de etapas invasoras iniciales, como también a la radio y quimioterapia en casos avanzados. Todo esto ha mejorado la atención asegurando un tiempo de latencia menor entre diagnóstico y tratamiento, como asimismo mejorando la calidad de ella con protocolos estandarizados y aporte de las tecnologías actuales.

La $8^{\underline{a}}$ Región presentó una tasa de mortalidad por CACU de 10,9/100.000 mujeres el año 2005, mayor que el promedio nacional. Esto nos motivó a revisar y comunicar nuestra realidad hospitalaria con el panorama completo de la distribución de casos de CACU, desde los casos diagnosticados en la UPC, las atendidas en el servicio de Ginecología como en el servicio de Oncología del Hospital Guillermo Grant B. de Concepción.

Planteamos esta inquietud inicial, acorde con las directrices ministeriales respecto a la necesidad de contar con un registro hospitalario confiable, que a futuro sea reconocido internacionalmente, que favorezca y permita la investigación local, produciendo información periódica de la etapa al momento del diagnóstico, como también el tratamiento y la supervivencia, apuntando a optimizar y cubrir necesidades de la administración hospitalaria, previniendo necesidades futuras y contribuyendo al registro poblacional de la patología.

\section{MATERIAL Y MÉTODO}

Estudio de corte transversal en que se analizan los datos de 1.131 mujeres con CACU, consultantes en la UPC, con lesiones in situ e invasor, de las sometidas a cirugía en la Unidad de Ginecología y de las atendidas en el Servicio de Oncología Hospital Guillermo Grant B. de Concepción, entre los años 1999 - 2005.

Tanto en la UPC como en el Servicio de Oncolo- gía hubo casos de mujeres derivadas de otros servicios de salud, por lo que estos resultados son del período de estudio en el Hospital Guillermo Grant B. La clasificación se realizó en base al informe histopatológico para lesiones in situ y de invasión temprana. Los casos de lesiones avanzadas fueron consignados de acuerdo al diagnóstico clínico emitido por el comité onco-ginecológico. De 609 casos de cáncer avanzado, 25 (4,1\%) no poseen diagnóstico de etapa clínica. Se aplicó la clasificación FIGO del 2004. Para el análisis de la información se utilizó el paquete estadístico SPSS 16. Los resultados se expresan en frecuencias y promedios.

\section{RESULTADOS}

En el período estudiado hubo 1.131 mujeres con CACU, $522(46,1 \%)$ presentaron lesiones en Etapa 0 , con una edad promedio de 34,9 años (rango: 1675 años), y 609 con cáncer invasor, con una edad promedio de 51,2 años (rango: 19-95 años). Hubo 74 casos con informes de CACU Etapa la y lb1 sin tumor clínico, diagnosticados a través de estudio histológico con asa electroquirúrgica.

La distribución de la etapificación de los 609 casos de CACU invasor se presenta en la Tabla I. Se destaca que los casos se concentran en las etapas lb $(28,1)$, Ilb $(26,4 \%)$ y III b $(24,3 \%)$.

En Tabla II se presenta la distribución por edad de las pacientes con CIS y cáncer invasor. Se destaca que el $77,6 \%$ de las lesiones intraepiteliales corresponde al rango de edad entre 25-44 años, mientras que para cáncer invasor el $62,2 \%$ se concentra en mayores de 45 años.

Tabla I

\section{DISTRIBUCIÓN DE 609 CASOS DE CÁNCER CERVICOUTERINO INVASOR SEGÚN FIGO,} 1999-2005

\begin{tabular}{lrrcc}
\hline $\begin{array}{l}\text { ETAPA } \\
\text { FIGO }\end{array}$ & \multicolumn{1}{c}{$\mathrm{n}$} & $\%$ & $\begin{array}{c}\text { Edad } \\
\text { promedio }\end{array}$ & Rango \\
\hline I a & 52 & 8,53 & 40,6 & $22-64$ \\
I b & 171 & 28,07 & 49,2 & $19-84$ \\
II a & 33 & 5,41 & 59,2 & $31-86$ \\
II b & 161 & 26,43 & 51,4 & $22-95$ \\
III a & 11 & 1,80 & 57,5 & $39-77$ \\
III b & 148 & 24,30 & 54,9 & $31-82$ \\
IV a & 6 & 0,98 & 53,5 & $48-77$ \\
IV b & 2 & 0,32 & 42,5 & $33-52$ \\
Sin evaluar & 25 & 4,10 & - & - \\
\hline
\end{tabular}


Tabla II

\section{RANGO Y PORCENTAJES DE EDAD EN CIS Y CANCER INVASOR}

\begin{tabular}{|c|c|c|c|c|}
\hline \multirow[b]{2}{*}{$\begin{array}{l}\text { Rango } \\
\text { (años) }\end{array}$} & \multicolumn{2}{|c|}{ Cáncer in situ } & \multicolumn{2}{|c|}{ Cáncer Invasor } \\
\hline & $\mathrm{n}$ & $\%$ & $\mathrm{n}$ & $\%$ \\
\hline $15-24$ & 47 & 9,0 & 4 & 0,65 \\
\hline $25-34$ & 212 & 40,6 & 42 & 6,89 \\
\hline $35-44$ & 193 & 37,0 & 159 & 26,10 \\
\hline $45-54$ & 59 & 11,3 & 144 & 23,64 \\
\hline $55-64$ & 7 & 1,3 & 118 & 19,37 \\
\hline 65 & 4 & 0,8 & 117 & 19,21 \\
\hline Ignorado & - & - & 25 & 4,10 \\
\hline TOTAL & 522 & 100 & 609 & 100 \\
\hline
\end{tabular}

\section{DISCUSIÓN}

Los resultados publicados de la distribución de casos de acuerdo a la etapa FIGO, generalmente se refieren a las etapas invasoras. En nuestra casuística la Etapa 0 (CIS), representa $46,1 \%$ del total, que sumados a los casos clasificados como Etapa la, resulta ser el $50,7 \%$ del total de casos, que consideramos como diagnóstico precoz, lo que está relacionado con el aumento de cobertura de los programas, que garantiza progresivamente el éxito de ellos $(1,2,9,10)$.

La Unidad de Cáncer del Ministerio de Salud de Chile, reportó para el año 1990, un total nacional de 863 casos con $27 \%$ de mujeres clasificadas en Etapa I. En el 2003 aumentó a $48 \%$ de un total de 1.043 casos. Asimismo el formulario de evaluación del año 2006, con un total de 935 casos, muestra $50,4 \%$ de mujeres consignadas en Etapa I (3), similar a publicaciones internacionales recientes de países europeos $(11,12)$.

Nuestros resultados tomando los 609 casos de cáncer invasor, que son el promedio de los 7 años de estudio, muestran $36,6 \%$ de mujeres en Etapa I, que disminuyen progresivamente a $31,8 \%, 26,1 \%$ y $1,3 \%$ respectivamente en etapa II, III y IV, respectivamente. Esta tendencia a disminuir es similar a lo reportado por el MINSAL, pero superiores a las estadísticas nacionales de los años 2003 y 2006 (3). Esta observación puede estar condicionada por las muestras diferentes, los períodos analizados (1 año versus 7 años), por la diferente incidencia del CACU a lo largo del país, por las diferencias de cobertura y cumplimiento del programa, y por varia- bles propias de la enfermedad.

El análisis comparativo con países con alta incidencia de la enfermedad, de acuerdo a la distribución de casos en etapa clínica invasora, revela que nuestros casos se concentran mayoritariamente en etapas iniciales, con mayor porcentaje de diagnóstico precoz, que apunta al éxito de nuestro programa $(1,2,10,11,13,14)$. Asimismo cuando comparamos las lesiones de CIS y microinvasoras, que representan en nuestro estudio al $50,7 \%$, es comparativamente muy superior (15).

La disminución progresiva del número de casos a medida que se avanza en las etapas invasoras, es global por etapa, disminuyendo progresivamente de la Etapa I a la IV. Cuando aplicamos la clasificación de acuerdo a las subetapas a y b, la distribución se concentra mayoritariamente en las sub etapas $b$.

La clasificación tiene relación con la historia natural de propagación de la enfermedad y el pronóstico que deriva de ella, es de suponer que deben existir factores que determinan el momento del diagnóstico de etapa clínica al inicio, como condiciones específicas del huésped, biológicas y socioculturales, que podrían explicar esta observación que revela además, la complejidad para mejorar los índices de morbimortalidad de la enfermedad, que dependen de estas circunstancias.

El porcentaje de mujeres menores de 25 años con CIS, en nuestro estudio fue $9 \%$. Esta cifra refrenda la tendencia de aumento de lesiones en mujeres jóvenes, descrita en la literatura $(16,17)$. Por el otro lado, el $19,2 \%$ de mujeres con cáncer invasor resultaron mayores de 65 años, revelando disminución o falta de control en el período post reproductivo, con una concentración de casos de mujeres de mayor edad en etapas mas avanzadas.

Estos resultados apuntan a plantear un análisis del costo-beneficio de incluir en programas de detección al grupo de mujeres menores de 25 años y a mayores de 65 años, que actualmente no las incluye y que es la interrogante planteada a la luz de resultados similares reportada también por otros autores $(1,16,17)$.

En nuestra casuística hubo sólo 4 mujeres menores de 25 años con cáncer invasor $(0,65 \%)$. Destacamos uno de estos casos que corresponde a una adolescente de 19 años de procedencia rural, que debuta con etapa Ib1, lesión escamosa, moderadamente diferenciada, operada, resultando con metástasis ganglionares y compromiso microscópico de parametrios. Posteriormente irradiada, que finalmente fallece al año por progresión de la enfermedad. En este caso claramente no existió el tiempo de evolución declarado para mantener y 
progresar a lesiones neoplásicas del cérvix, acorde con el conocimiento de la historia natural de propagación de la enfermedad, reflejando lo complejo del enfrentamiento a la patología neoplásica y la trascendencia específica que implica la relación de ella con el huésped.

\section{CONCLUSIONES}

La aplicación del Programa Nacional de pesquisa precoz del cáncer cérvico-uterino permitió que el $46,1 \%$ de los casos correspondan a diagnóstico precoz, contribuyendo así a la tendencia nacional de disminución de la tasa de mortalidad por esta patología. De acuerdo con nuestros resultados es necesario analizar el costo-beneficio de incluir mujeres menores de 25 años y mayores de 65 en el programa de pesquisa precoz del cáncer cérvicouterino.

\section{BIBLIOGRAFÍA}

1. Simcock B, Sykes P, Laney M. The impact of the National Cervical Screening Programme on the presentation of cancer of the cervix in Canterbury. N Z Med J 2001;114(1138):378-80.

2. Karim-Kos HE, de Vries E, Suerjomataran I, Lemmens V, Siesling S, Coerbergh JW. Recent trends of cancer in Europe: a combined approach of incidence, survival and mortality for 17 cancer sites since the 1990s. Eur J Cancer 2008;44(10):1345-89.

3. Ministerio de Salud. Guía Clínica Cáncer Cérvicouterino. Hallado en: http://www.redsalud.gov.cl/archivos/ guiasges/CancerCervicouterino.pdf DEIS:Servicios de Salud/Unidad de Cancer/Minsal/matrona M. Prieto/ Dr. E. Suarez

4. Suárez E, Prieto M, Rojas I, et al. Programa Nacional de Cáncer Cervicouterino. Rev Chil Obstet Ginecol 2001;66(6):480-91.

5. Donoso E, Cuello M, Villarroel L. Reducción de la mortalidad por cáncer cervicouterino en Chile, 1990-2003. Rev Chil Obstet Ginecol 2006;71(5):307-12.

6. Dobs SP, Asmussen T, Nunns D, Hollingworth J, Brown LJ, Ireland D. Does histological incomplete excision of cervical intraepithelial neoplasia following large loop excision of transformation zone increase recurrence rates? A six year cytological follow up. BJOG 2001;107(7):1298-301.

7. Numnum TM, Kirby TO, Leath CA 3rd, Huh WK, Alvarez RD, Straughn JM Jr. A prospective evaluation of "see and treat" in women with HSIL Pap smear results: is this an appropriate strategy? J Low Genit Tract Dis 2005;9(1):2-6.

8. Nazzal O, Reinero M, Abarzúa A, et al. Patología preinvasora del cérvix. Rev Chil Obstet Ginecol 2003;68(3):18996.

9. Haldorsen T, Skare GB, Steen R, Thoresen SO. Cervical cancer after 10 years of nationally coordinated screening. Tidsskr Nor Laegeforen 2008;128(6):6825.

10. Simon $P$, Dupond I. Screening for cervical cancer. Rev Med Brux 2006;27(4):S218-20.

11. Papanikolaou A, Kalogiannidis I, Misailidou D, Goutzioulis M, Stamatopoulos P, Makedos A, Vatopoulou A, Makedos G.Results on the treatment of uterine cervix cancer: ten years experience. Eur J Gynaecol Oncol 2006;27(6):607-10.

12. Hagen B, Skjeldestad FE, Halvorsen $T$, et al. Primary treatment of cervical carcinoma. Ten years experience from one Norwegian health region. Acta Obstet Gynecol Scand 2000;79(12):1093-9.

13. Chirenje ZM, Rusakaniko S, Akino V, Mlingo M. A review of cervical cancer patients presenting in Harare and Parirenyatwa Hospitals in 1998. Cent Afr J Med 2000;46(10):264-7.

14. Jain VS, Singh KK, Shrivastava R, Saumsundaran $\mathrm{KV}$, Sarje MR. Radical radiotherapy treatment of carcinoma of the uterine cervix: outcome in patients treated at a rural center in India. J Cancer Res Ther 2007;3(4):211-7.

15. Badar F, Anwar N, Meerza F, Sultan F. Cervical carcinoma in a Muslim community. Asian Pac J Cancer Prev 2007;8(1):24-6.

16. Nair MS, Bhandari HM, Nordin Al. Cervical cancer in women aged less than 25: East Kent experience. J Obstet Gynaecol 2007;27(7):706-8.

17. Bielsa-Lasota $M$, Inghelmann $R$, van de Poll-Franse I, Capocaccia R. Trends in cervical cancer survival in Europe, 1983-1994: a population-based study. Gynecol Oncol 2007;105(3):609-19. 